\title{
Phenotypic characterization of type II collagen-induced arthritis in Wistar rats
}

\author{
HOU-PAN SONG ${ }^{1 *}$, XIN LI $^{1 *}{ }^{*}$ RONG YU ${ }^{2}$, GUANG ZENG $^{1}$, ZHEN-YI YUAN $^{2}$, \\ WEI WANG ${ }^{3}$, HUI-YONG HUANG ${ }^{1}$ and XIONG CAI ${ }^{1,2}$ \\ ${ }^{1}$ Hunan Provincial Key Laboratory of Diagnostics in Chinese Medicine; ${ }^{2}$ Hunan Department of \\ Education's Key Laboratory of Translational Chinese Medicine; ${ }^{3}$ School of Pharmaceutical Sciences, \\ Hunan University of Chinese Medicine, Changsha, Hunan 410007, P.R. China
}

Received August 9, 2014; Accepted May 1, 2015

DOI: $10.3892 /$ etm.2015.2667

\begin{abstract}
The aim of the present study was to determine a more specific, efficient and simple method for the induction of collagen-induced arthritis (CIA) in rats. Different strains of rats were injected at the base of the tail with bovine type II collagen (CII) emulsified in incomplete Freund's adjuvant (IFA). The onset and severity of arthritis were evaluated by clinical assessment. The established CIA model was analyzed using a comprehensive examination of clinical, hematological, histological and radiological parameters. The results demonstrated that Wistar rats were the most susceptible strain to CIA followed by Wistar Furth rats, with Sprague Dawley rats being the least susceptible. Following primary and booster immunization, female Wistar rats developed severe arthritis, with an incidence of $>83 \%$ and low variability in clinical signs. The development of arthritis was accompanied by a significantly elevated erythrocyte sedimentation rate compared with that in the control rats. The radiographic examination revealed bone matrix resorption, considerable soft tissue swelling, periosteal new bone formation and bone erosion in the arthritic joints of the CIA rats. Histopathologically, the synovial joints of CIA rats were characterized by synovial hyperplasia, pannus formation, marked cellular infiltration, bone and cartilage
\end{abstract}

Correspondence to: Professor Xiong Cai or Professor Hui-Yong Huang, Hunan Provincial Key Laboratory of Diagnostics in Chinese Medicine, Hunan University of Chinese Medicine, 113C Shaoshan Road, Changsha, Hunan 410007, P.R. China

E-mail: caix12@qq.com

E-mail: huanghy@126.com

*Contributed equally

Abbreviations: CIA, collagen-induced arthritis; CII, type II collagen; IFA, incomplete Freund's adjuvant; SD, Sprague Dawley; CFA, complete Freund's adjuvant; ESR, erythrocyte sedimentation rate; RA, rheumatoid arthritis; AIA, adjuvant-induced arthritis

Key words: collagen-induced arthritis, bovine type II collagen, incomplete Freund's adjuvant, Wistar rats erosion and narrowing of the joint space. The administration of an intradermal injection of only $200 \mu \mathrm{g}$ bovine CII emulsified in IFA at the base of the tail therefore leads to the successful development of a CIA rat model. This well-characterized CIA rat model could be specifically used to study the pathophysiology of human rheumatoid arthritis as well as to test and develop anti-arthritic agents for humans.

\section{Introduction}

Collagen-induced arthritis (CIA) was first described by Trentham et al (1) in both outbred and inbred rat strains following immunization with type II collagen (CII), the major constituent protein of articular cartilage. CIA, an experimental model of autoimmune arthritis, has been shown to exhibit similar histological, immunological and clinical characteristics and genetic linkage to human rheumatoid arthritis (RA) $(1,2)$. CIA has therefore been widely used in laboratories worldwide in studies focusing on the pathogenesis of RA and the preclinical evaluation of novel therapeutics for the disease (2,3).

The immune response to CII and subsequent development of arthritis in rats is linked to the RT1 locus of the rat major histocompatibility complex (4). The arthritogenic potency of CII from different species used for immunization varies; in general, porcine CII is the most potent, followed by bovine and chicken CII (2). As described in numerous studies from Eastern and Southern Asia, CIA is induced in rats predominantly by immunizing the animals with either chicken or bovine CII emulsified in complete Freund's adjuvant (CFA) containing heat-killed Bacillus Calmette-Guérin in liquid paraffin (5-8). The injection is administered in either the subplantar tissues of one of the hind paws $(5)$, at the base of the tail $(7,8)$ or at both the base of the tail and the back (6). These immunization protocols, however, are associated with several problems, one of which is that CFA alone can induce adjuvant arthritis (AIA) in rats $(9,10)$. The use of CFA for the preparation of CII emulsion could therefore produce an immune response to antigens other than CII in the immunized rats. In addition, the injection of the rat footpads with a high concentration of the CII/CFA emulsion puts the injected paws at risk of a severe infection from bacteria contained in the CFA, which could eventually 
result in the failure of the experiments. Furthermore, intradermal injection at multiple locations at once, such as the base of the tail and the back of the rat, is not only time-consuming and inconvenient to perform, but also requires larger amounts of CII emulsion (0.5-1 ml) for the induction of arthritis.

The aim of the present study was to determine a more simple, specific and efficient method for the induction of CIA in rats. Incomplete Freund's adjuvant (IFA) was used instead of CFA to emulsify bovine CII, and the CII/IFA emulsion was intradermally injected at the base of the tail, with a booster injection administered on day 7 after primary immunization. Using this immunization method, different strains of rats of both genders were compared according to the susceptibility they exhibited to CIA. Finally, the most successfully established CIA model was characterized in terms of clinical, hematological, histopathological and radiological features of human RA, in order to provide scientific evidence for the reliability of the model and for its specific use in studies on the etiopathogenetic mechanisms of RA and for the development of novel anti-arthritic drugs.

\section{Materials and methods}

Preparation of collagen emulsion. A CII/IFA emulsion $(1 \mathrm{mg} / \mathrm{ml})$ was freshly prepared as previously described $(2,11)$. Briefly, an equal volume of $2 \mathrm{mg} / \mathrm{ml}$ bovine CII in $0.05 \mathrm{M}$ acetic acid and IFA (Chondrex, Inc., Redmond, WA, USA) was thoroughly mixed in an ice water bath, using a homogenizer, to produce a stable emulsion that would remain as a solid clump and not dissipate in the water.

Development of CIA. Specific pathogen-free outbred male and female Wistar, Wistar Furth and Sprague Dawley (SD) rats (Vital River Laboratory Animal Technology Co., Ltd., Beijing, China), aged 6-7 weeks, were used. The rats were housed 4 per cage in rooms maintained at $20 \pm 0.5^{\circ} \mathrm{C}$ with a 12 -h light/dark cycle. The animals had free access to food and water and were acclimated to their surroundings for 1 week prior to the initiation of the experiments.

The CIA model was induced in the rats as previously described $(11,12)$. CIA was induced in 6 rats per group to define the most susceptible strain to CIA, and in 8 rats per group to characterize the features of CIA in female Wistar rats. Briefly, the rats were intradermally injected in two sites at the base of the tail with a total of $0.1 \mathrm{ml} \mathrm{CII/IFA} \mathrm{emulsion.} \mathrm{On} \mathrm{day} 7$ after primary immunization, a booster injection of $0.1 \mathrm{ml} \mathrm{CII/IFA}$ emulsion was administered to all rats. All experimental protocols regarding the animals and their care were approved by the Institutional Animal Care and Use Committee of Hunan University of Chinese Medicine (Changsha, China) and carried out in strict accordance with the Guide for the Care and Use of Laboratory Animals (National Institutes of Health, Bethesda, MD, USA).

Clinical evaluation of the development of CIA. Arthritic signs in the paws of the rats, characterized by edema and erythema, were inspected daily following the primary CII/IFA injection, and the onset of arthritis was monitored. To evaluate the incidence and severity of the arthritis, lesions on all 4 paws of each rat (i.e. the arthritic signs) were graded by two independent investigators using an arthritic scoring system. Lesions on all four paws of each rat (i.e., the arthritic signs) were graded from 0 to 4 according to the extent of edema and erythema of the periarticular tissues; 16 was the potential maximum of the combined arthritic scores per animal (Rosloniec, Cai). The severity scores were defined as follows: 0 , No evidence of erythema and swelling; 1, erythema and mild swelling confined to the mid-foot (tarsals) or ankle joint; 2, erythema and mild swelling extending from the ankle to the foot; 3 , erythema and moderate swelling extending from the ankle to the metatarsal joints; and 4, erythema and severe swelling encompass the ankle, foot and digits. The hind paw volumes were measured every 3 days using a plethysmometer chamber (UGO SLR, Comerio, Italy), beginning on day 9 after the primary immunization $(11,12)$. In addition, the body weight of the rats was monitored every 3 days.

Determination of erythrocyte sedimentation rate (ESR). Blood samples were collected from the veins of the rat tails $0,6,12$, 18, 24 and 30 days after the CII/IFA injection for laboratory testing. The ESR was determined by a modified method as previously described (13).

Radiological and histopathological studies. Upon conclusion of the experiments, the rats were sacrificed by diethyl ether asphyxiation. The hind paws were radiographed and then histopathologically analyzed as previously described $(11,12)$.

Statistical analysis. All statistical analyses were performed using SPSS software, version 20.0 (SPSS, Inc., Chicago, IL, USA). Data are presented as the mean \pm standard error of the mean. The differences between the groups were analyzed using the Student's t-test. A two-tailed $\mathrm{P}<0.05$ was considered to indicate a statistically significant difference.

\section{Results}

Susceptibility of different rat strains to CIA. As shown in Table I, neither male nor female SD rats were susceptible to the immunization with bovine CII/IFA at the base of the tail, while Wistar Furth rats were moderately responsive to CIA. By contrast, female Wistar rats were greatly susceptible to CIA, developing severe arthritis with an incidence of $>83 \%$.

Clinical progression of CIA in female Wistar rats. Following immunization with the CII/IFA emulsion at the base of the tail, the onset of arthritis occurred in female Wistar rats around days 10 to 16. In contrast to the control rats (Fig. 1A), the CIA rats exhibited conspicuous inflammatory lesions, characterized by edema and erythema in the paws (Fig. 1B). Fig. 2 shows the disease progression of the CII-induced arthritic lesions in female Wistar rats. From day 12 onwards, the arthritic score (Fig. 2A) and hind paw volume (Fig. 2B) were significantly increased in the CIA rats as compared with those in the control rats; the parameters reached a plateau on days 18-21 and decreased gradually thereafter. Marked body weight loss was also observed in the CIA rats as compared with the control rats (Fig. 2C). With regard to the disease progression, the ESR was significantly elevated in the CIA 
Table I. Susceptibility of different rat strains to collagen II-induced arthritis.

\begin{tabular}{|c|c|c|c|c|}
\hline Strain & Gender & Incidence $^{\mathrm{a}}, \%$ & Onset day ${ }^{\mathrm{b}}$, range (mean) & Severity $^{\mathrm{c}}$ \\
\hline \multirow[t]{2}{*}{ Sprague Dawley } & Male & 0.0 & N/A & N/A \\
\hline & Female & 0.0 & N/A & N/A \\
\hline \multirow[t]{2}{*}{ Wistar } & Male & 0.0 & $\mathrm{~N} / \mathrm{A}$ & N/A \\
\hline & Female & 83.3 & $10-16(13)$ & 7.6 \\
\hline \multirow[t]{2}{*}{ Wistar Furth } & Male & 50.0 & $10-18(14)$ & 2.5 \\
\hline & Female & 33.3 & $9-15(12)$ & 1.7 \\
\hline
\end{tabular}

${ }^{a} \mathrm{n}=6$ /group. ${ }^{\mathrm{b}}$ Mean onset day was calculated by averaging the onset day of each rat per group. ${ }^{\mathrm{c}}$ Severity is expressed as the mean of the highest arthritic score of each rat per group. N/A, not applicable.
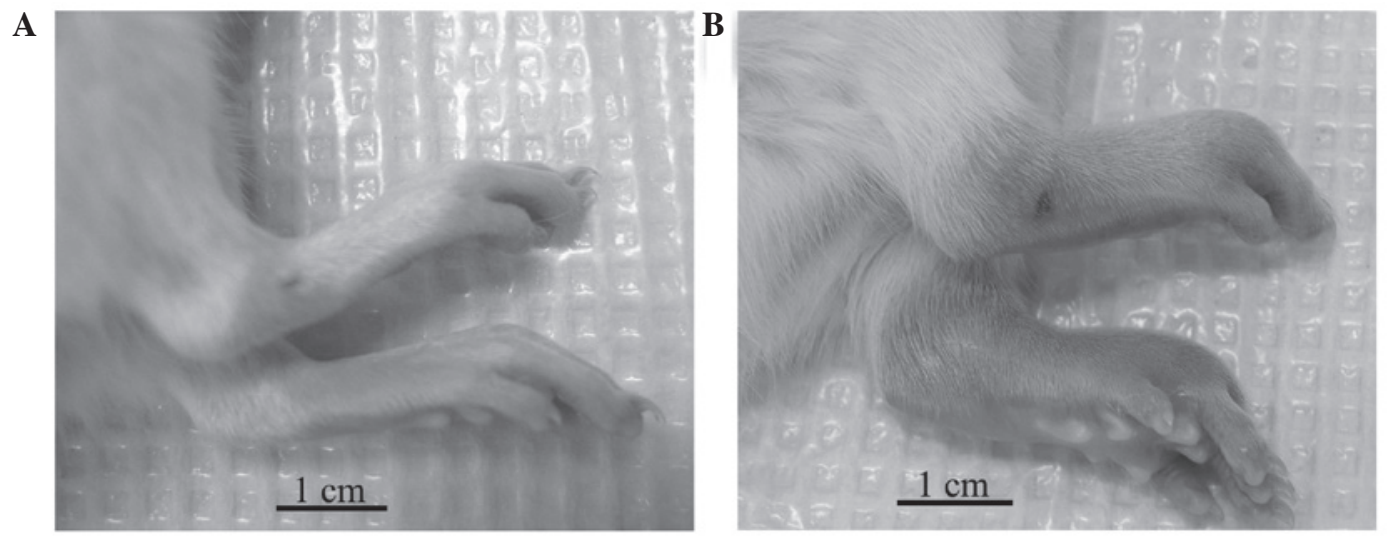

Figure 1. Clinical signs of CIA in the hind paws of female Wistar rats. (A and B) Representative images of the hind paws from (A) control and (B) CIA rats taken on day 15 after the primary injection of type II collagen emulsified in incomplete Freund's adjuvant. Intense edema and erythema were observed in the hind paws of the CIA rats compared with the control rats. CIA, collagen-induced arthritis.

A

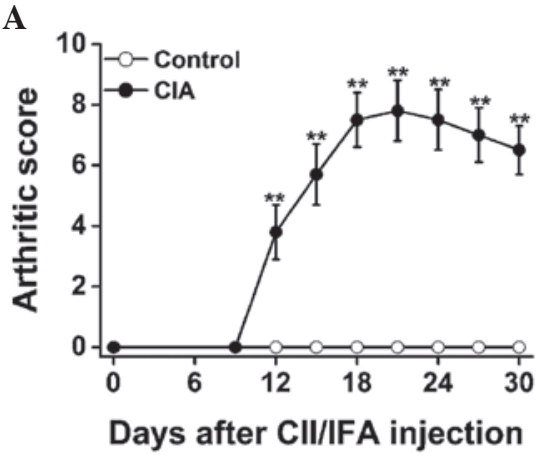

C

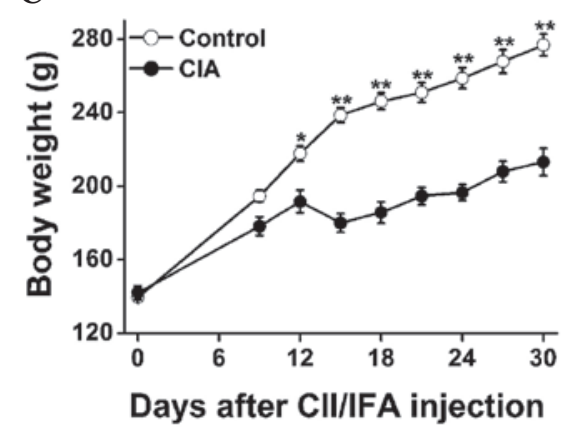

B

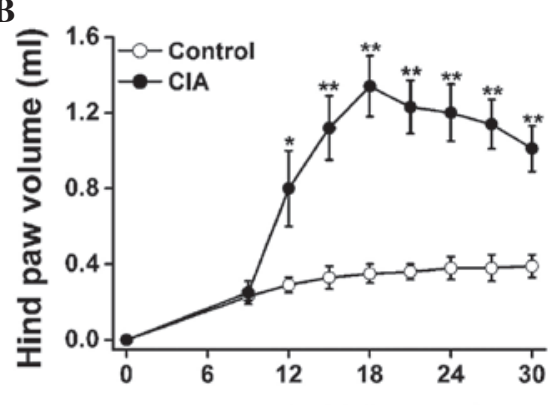

Days after CII/IFA injection

D

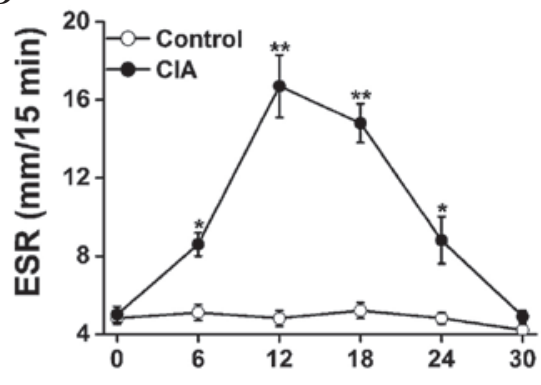

Days after CII/IFA injection

Figure 2. Clinical progression of CIA in female Wistar rats during a 30-day course: (A) Arthritic score, (B) hind paw volume, (C) body weight and (D) ESR Data are presented as the mean \pm standard error of the mean $(n=8)$. " $\mathrm{P}<0.05$ and ${ }^{* *} \mathrm{P}<0.01$ versus control rats. CII, type II collagen; IFA, incomplete Freund's adjuvant; CIA, collagen-induced arthritis; ESR, erythrocyte sedimentation rate. 
A

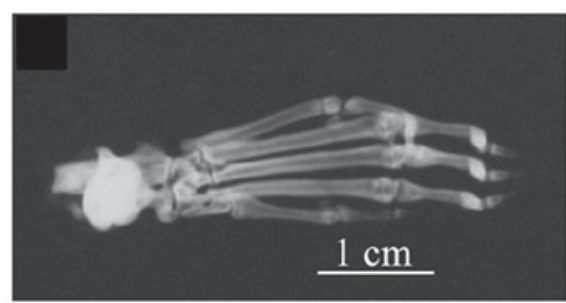

C

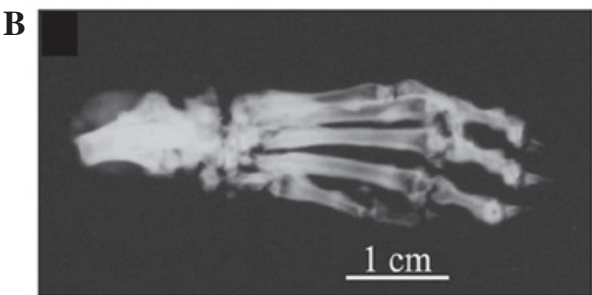

$\square$ Control

$\square$ CIA

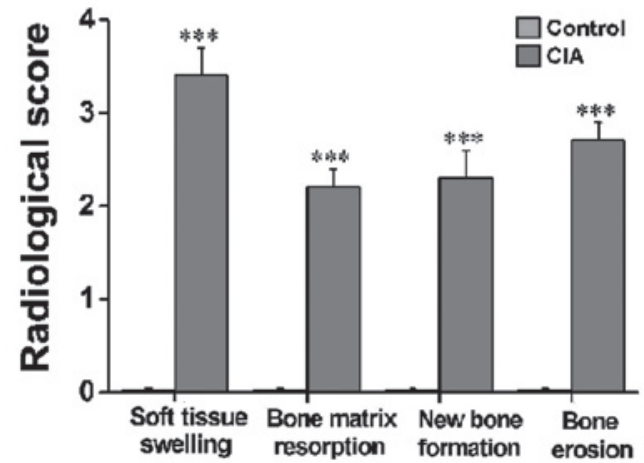

Figure 3. Radiographic evidence of CIA in the hind paws of female Wistar rats. (A and B) Representative radiographs taken on day 30 after the primary injection of type II collagen emulsified in incomplete Freund's adjuvant. Compared with the hind paws of the (A) control rats, the hind paws of the (B) CIA rats exhibited severe soft tissue swelling, bone matrix resorption, periosteal new bone formation and bone erosion. (C) Radiological analysis showed significant differences in these radiological parameters between the two groups of rats. Data are presented as the mean \pm standard error of the mean $(\mathrm{n}=8)$. ${ }^{* * * *} \mathrm{P}<0.001$ versus control rats. CIA, collagen-induced arthritis.

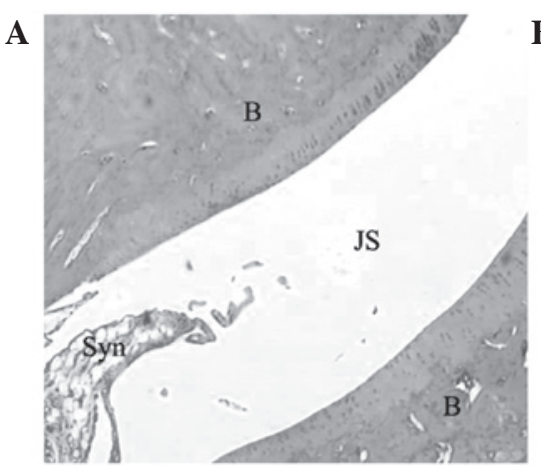

C

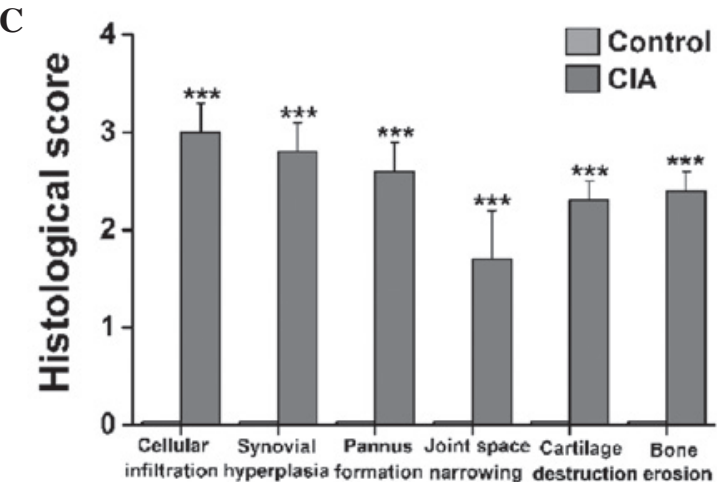

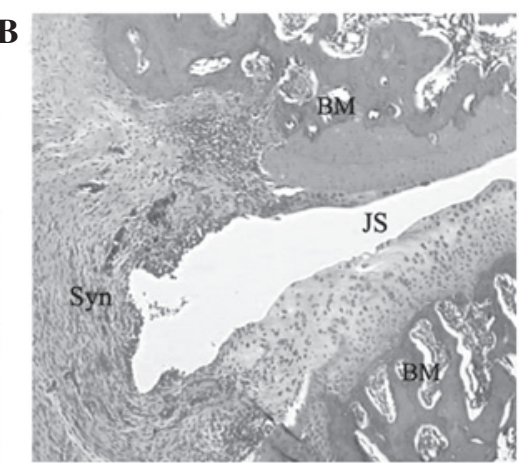

Control

CIA 
visible in the CIA rats. It was evident in the representative radiographs that the CIA rats (Fig. 3B) exhibited severe soft tissue swelling, a prominent decrease in bone density, notable destruction of bony structures and ossifications not contiguous with normal bone-line in the tarsal, metatarsal and interphalangeal regions as compared with the control rats (Fig. 3A). The radiological scoring of soft tissue swelling, bone matrix resorption, periosteal new bone formation and bone erosion showed significant differences between the CIA and control rats (Fig. 3C).

Histopathological analysis of CIA in female Wistar rats. As noted in the representative hematoxylin and eosin-stained sections of the ankle joints from the control (Fig. 4A) and CIA (Fig. 4B) rats, a clear space between the thin synovial membrane and the bones was found in the control rats (Fig. 4A), while extensive synovial proliferation, marked cellular infiltration and considerable pannus formation were observed in the CIA rats (Fig. 4B). The CIA rats also exhibited a marked loss of joint space and severe erosion of the bones and cartilage (Fig. 4B). Fig. 4C illustrates statistically significant differences in the histopathological scoring of cellular infiltration, joint space narrowing, cartilage destruction, bone erosion, synovial hyperplasia and pannus formation between the CIA and control rats $(\mathrm{P}<0.001)$.

\section{Discussion}

CIA is an experimental model of autoimmune-mediated arthritis that has been used worldwide in a wide variety of studies, including studies focusing on the understanding of the immunopathogenic mechanisms of arthritis, the analysis of genetic susceptibility factors and the preclinical development of novel therapeutic approaches. CIA can be induced in susceptible strains of rodents (rats and mice) and non-human primates by CII immunization $(1,2,14)$. With regard to the establishment of CIA in rats, which are generally susceptible to AIA, the use of potent immunoadjuvants (e.g. CFA) should be obviated. A more simple and efficient immunization method, which requires smaller amounts of CII emulsion, could be particularly advantageous for research purposes.

In the present study, the susceptibility of different rat strains to immunization with bovine CII emulsified in IFA at the base of the tail was in the following order: SD, Wistar Furth and Wistar (from lowest to highest, respectively) (Table I). SD rats were relatively resistant to CIA and Wistar Furth rats were exhibited a moderate response (Table I). By contrast, female Wistar rats were highly responsive to CIA and exhibited a high incidence and severity of arthritis (Table I). Following primary and booster immunization with a total of $0.2 \mathrm{ml} \mathrm{CII/IFA} \mathrm{emulsion} \mathrm{at} \mathrm{the} \mathrm{base} \mathrm{of}$ the tail, female Wistar rats developed pronounced arthritis with a high incidence and a low variability in clinical signs (Figs. 1 and 2).

In association with the development of arthritis, the ESR in the CIA rats was found to be markedly elevated between days 6 and 24 after CII/IFA immunization, as compared with that in the control rats (Fig. 2D). ESR is a common laboratory test used to aid the definitive diagnosis of RA, the assessment of the disease activity and its response to therapeutics. Studies have reported that the ESR is affected by increases in the plasma levels of acute-phase reactant proteins (e.g. fibrinogen, $\alpha$ - and $\beta$-globulin); consequently, the ESR is considered to reflect the acute-phase response to inflammatory diseases $(15,16)$. In this CIA model of female Wistar rats, the ESR peaked on day 12 and underwent a sharp decrease thereafter, which indicated a relatively early production and stimulation of acute-phase proteins during the progression of the disease (Fig. 2D).

RA is initially characterized by the presence of an immune-mediated inflammatory response of the synovial membrane, accompanied by diffuse infiltration of a variety of mononuclear cells and the formation of new vessels. Chronic inflammation and the abnormal proliferation of the synovial membrane ultimately form a destructive pannus, which progressively invades and destroys the cartilage and bone in joints over the course of the disease $(17,18)$. In patients with RA, distinct patterns of bone loss are observed, such as juxta-articular osteopenia adjacent to inflamed joints and the presence of focal erosion within the subchondral bone and at the joint margins in areas of direct pannus invasion $(19,20)$. Bone remodeling also occurs in the arthritic joints $(21,22)$. In the present CIA model of female Wistar rats, histological sections of the ankle joints revealed diffuse cellular infiltration, extensive synovial proliferation, the formation of erosive panni, notable narrowing of the joint space and marked cartilage and bone erosion (Fig. 4). The radiological examination also revealed distinct patterns of bone loss and erosion associated with periosteal new bone formation in the tarsal, metatarsal and interphalangeal regions (Fig. 3). The combination of pronounced bone matrix resorption and heterotopic new bone formation was strongly indicative of the presence of a disordered pattern of bone remodeling in the CIA rats. Thus, the findings in the female Wistar rat CIA model closely resemble the disease characteristics of human RA.

In conclusion, a more specific, simple and efficient method of establishing a CIA rat model delineating the development of human RA has been determined in the present study. An intradermal injection of only $200 \mu \mathrm{g}$ bovine CII emulsified in IFA at the base of the tail can successfully induce a pronounced autoimmune-mediated polyarthritis in female Wistar rats. This CIA rat model shows a high disease incidence and low variability in clinical signs and closely resembles its human counterpart, RA. This well-developed and well-characterized CIA model in female Wistar rats could therefore be specifically and efficiently used to study the pathological and immunological processes of RA, as well as to test and develop novel anti-arthritic agents.

\section{Acknowledgements}

This study was supported by grants from the National Natural Science Foundation of China (grant no. 81373540), the Key Science \& Research Program of Hunan Department of Science \& Technology (grant no. 2012TF-1005), the Hunan Chinese Medicine Science \& Research Key Project (grant no. 201308) and the Open-Ended Science Foundation of National Key Subject of Traditional Chinese Medicine Diagnostics (grant no. 2013ZYZD10, 2014-8). 


\section{References}

1. Trentham DE, Townes AS and Kang AH: Autoimmunity to type II collagen: An experimental model of arthritis. J Exp Med 146: 857-868, 1977.

2. Rosloniec EF, Cremer M,Kang A and Myers LK: Collagen-induced arthritis. Curr Protoc Immunol Chapter 15: Unit 15.5, 2001.

3. Billingham ME: Models of arthritis and the search for anti-arthritic drugs. Pharmacol Ther 21: 389-428, 1983.

4. Griffiths MM: Immunogenetics of collagen-induced arthritis in rats. Int Rev Immunol 4: 1-15, 1988.

5. Zhu L, Wei W and Zheng YQ: Effect and mechanism of action of total glucosides of paeony on synoviocytes from rats with collagen-induced arthritis. Yao Xue Xue Bao 41: 166-170, 2006 (In Chinese).

6. Wang Y, Zhao HY, Liu MJ, et al: Establishment of a rat model of rheumatoid arthritis with kidney deficiency syndrome. Zhong Xi Yi Jie He Xue Bao 9: 973-982, 2011 (In Chinese).

7. Lin CM, Gu J, Zhang Y, Shen LJ, Ma L, Ni J, Wang ZQ and $\mathrm{Wu} \mathrm{W}$ : Effect of UC-MSCs on inflammation and thrombosis of the rats with collagen type II induced arthritis. Zhonghua Xue Ye Xue Za Zhi 33: 215-219, 2012 (In Chinese).

8. Srivastava NK, Sharma S, Purusottam RN, et al: Abnormal lipid metabolism in collagen-induced arthritis rat model: In vitro, high resolution NMR spectroscopy based analysis. Indian J Exp Biol 52: 673-682, 2014.

9. Pearson CM: Experimental joint disease observations on adjuvant-induced arthritis. J Chronic Dis 16: 863-874, 1963.

10. Cai X, Wong YF, Zhou H, et al: Manipulation of the induction of adjuvant arthritis in Sprague Dawley rats. Inflamm Res 55: 368-377, 2006

11. Cai X, Zhou H, Wong YF, et al: Suppression of the onset and progression of collagen-induced arthritis in rats by QFGJS, a preparation from an anti-arthritic Chinese herbal formula. J Ethnopharmacol 110: 39-48, 2007.
12. Zhou H, Wong YF, Wang J, Cai X and Liu L: Sinomenine ameliorates arthritis via MMPs, TIMPs and cytokines in rats. Biochem Biophys Res Commun 376: 352-357, 2008.

13. Shen FY, Li X, Huang HY, et al: A simple, rapid and accurate method for determination of erythrocyte sedimentation rate using capillary tubes in experimental models of rodents. Zhong Guo Yao Li Xue Tong Bao 29: 1762-1765, 2013 (In Chinese).

14. Courtenay JS, Dallman MJ, Dayan AD, Martin A and Mosedale B: Immunisation against heterologous type II collagen induces arthritis in mice. Nature 283: 666-668, 1980.

15. Singh AS, Atam V, Yathish BE, Das L and Koonwar S: Role of erythrocyte sedimentation rate in ischemic stroke as an inflammatory marker of carotid atherosclerosis. J Neurosci Rural Pract 5: 40-45, 2014.

16. Solberg BL and Olson RJ: Clinical utility of the erythrocyte sedimentation rate: A case study. Clin Lab Sci 27: 72-77, 2014.

17. McGonagle D, Conaghan PG, O'Connor P, et al: The relationship between synovitis and bone changes in early untreated rheumatoid arthritis: A controlled magnetic resonance imaging study. Arthritis Rheum 42: 1706-1711, 1999.

18. Goldring SR and Gravallese EM: Pathogenesis of bone erosions in rheumatoid arthritis. Curr Opin Rheumatol 12: 195-199, 2000.

19. Gravallese EM and Goldring SR: Cellular mechanisms and the role of cytokines in bone erosions in rheumatoid arthritis. Arthritis Rheum 43: 2143-2151, 2000.

20. Smolen JS and Steiner G: Therapeutic strategies for rheumatoid arthritis. Nat Rev Drug Discov 2: 473-488, 2003.

21. Deodhar AA and Woolf AD: Bone mass measurement and bone metabolism in rheumatoid arthritis: A review. Br J Rheumatol 35: 309-322, 1996.

22. Fardellone P, Séjourné A, Paccou J and Goëb V: Bone remodelling markers in rheumatoid arthritis. Mediators Inflamm 2014: $484280,2014$. 